\title{
Spiral galaxies with large optical warps
}

\author{
V. Reshetnikov ${ }^{1,2}$ and F. Combes ${ }^{2}$ \\ 1 Astronomical Institute of St. Petersburg State University, 198904 St. Petersburg, Russia \\ 2 DEMIRM, Observatoire de Paris, 61 Av. de l'Observatoire, 75014 Paris, France
}

Received February 16; accepted June 1, 1999

\begin{abstract}
As a result of our statistical study of 540 edgeon galaxies, we present here the images and preliminary statistical analysis of a sub-sample of 60 galaxies, that were selected to be S-type warped spirals. Computing the average volumic density of galaxies from available redshift surveys, a first analysis suggests that warped galaxies are found in denser environments. Only the clearest and strongest warps have been extracted here, and therefore this sample of 60 objects gather the best candidates for future HI or optical works on galaxy warps.
\end{abstract}

Key words: galaxies: evolution; interactions; spiral; structure

\section{Introduction}

The majority of spiral galaxies have a warped plane, as has been revealed in the neutral gas extended component, through HI-21 cm observations (e.g. Bosma 1981; Briggs 1990), and in a lesser extent through optical observations (Sanchez-Saavedra et al. 1990; Reshetnikov \& Combes 1998). This dynamical feature raises the problem of its origin and maintenance, and the numerous mechanisms that have been proposed and explored have not yet given a definitive and satisfactory answer (e.g. the review by Binney 1992).

Differential precession should be very quick to wrap up any warp perturbation even in the outer parts of the galaxies (Kahn \& Woltjer 1959), unless the potential is nearly spherical (Tubbs \& Sanders 1979). But most warps are observed while the disk is still a significant part of the potential, which cannot therfore be spherical. It has been shown that coherent bending modes cannot be sustained, since the oscillations spectrum is continuous, for realistic disks that have no sharp edges (Hunter

Send offprint requests to: F. Combes

(e-mail: bottaro@obspm.fr)
\& Toomre 1969). Models then tried to consider a nonspherical dark halo, misaligned with the inner visible disk of the galaxy (Sparke 1984; Sparke \& Casertano 1988; Dubinski \& Kuijken 1995). However, these structures can only be transient, since the inner disk is bound to align with the dark halo (New et al. 1998; Binney et al. 1998). Alternatively, the warp could be the consequence of continuous accretion of gas with a slewed angular momentum, due to cosmic infall, as suggested by Ostriker \& Binney (1989) and Binney (1992). It is not excluded either that a large part of warps are due to interactions or mergers: the prototypical warped galaxy NGC 5907 (Sancisi 1976) that was long thought isolated, might have experienced a minor merger recently (Lequeux et al. 1998), and is currently interacting with two dwarf companions (Shang et al. 1998).

To progress about the puzzle of the origin of warps, it is important to have a sample of optically strongly warped galaxies, to perform new observations and statistical studies. Recently, we have presented a survey of optical warps in a sample of 540 galaxies, about 5 times larger than the previous samples (Reshetnikov \& Combes 1998). The galaxies were selected from the Flat Galaxy Catalogue of Karachentsev et al. (1993) (FGC) and we studied their optical images extracted from the Digitized Sky Surveys ${ }^{1}$. We identified three classes of galaxies, those without observable warps (30\%), and those with U-shaped (37\%) and S-shaped $(33 \%)$ warps. We have considered the artefacts due to projection effects, that could be severe in nearly edge-on galaxies, when there are spiral arms or $m=2$ perturbations. Through numerical simulations, it was found that the U-shape are more affected by projection effects, but that no more than $15 \%$ of S-shape warps could be geometrical artefacts. On the other hand, intrinsic warps could be missed through projection effects (but no more than 20\%).

We therefore select a sample of 60 S-shape warped galaxies, the strongest and clearest among the 174 found.

1 The Digitized Sky Surveys were produced at the Space Telescope Science Institute under U.S. Government grant NAG $\mathrm{W}-2166$. 


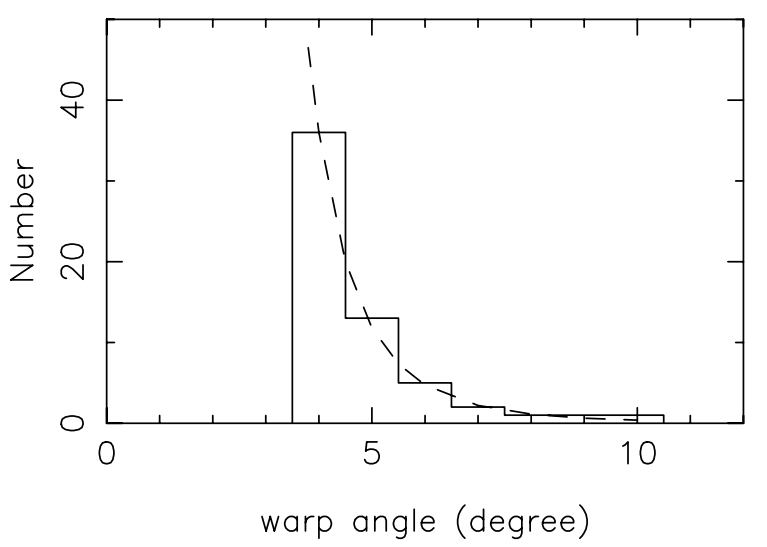

Fig. 1. Distribution of the observed warp angles. Dashed line shows $\psi^{-5}$ law

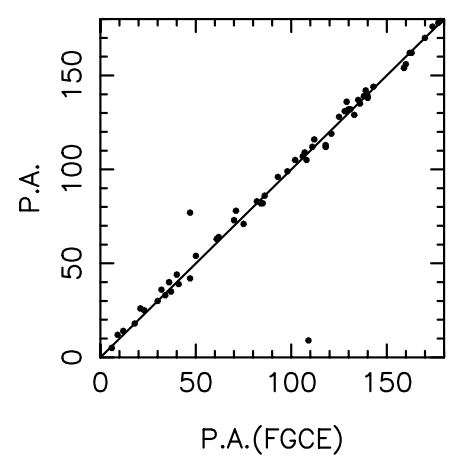

Fig. 2. Comparison between position angles measurements (in degrees) in our work (PA) and FGCE - PA (FGCE). The solid line shows equality

The selection is subjective, based on isophotal maps from the DSS. This sample should be a suitable material for future detailed HI and optical works on galaxy warps.

\section{The sample and statistics}

Table 1 presents extraction from the Southern Extention of FGC (Karachentsev et al. 1993, FGCE) for the galaxies with large S-type warps. The sample is limited by coordinates $0.0 \leq \alpha(1950) \leq 14.0, \delta(1950) \leq-17.5$. The columns are as follows: galaxy FGCE, PGC and ESO number; right ascension and declination for the epoch 1950.0; $B$ magnitude $\left(\mathrm{NED}^{2}\right)$; heliocentric radial velocity (NED); major and minor diameters measured on blue films (in arcmin); morphological type; warp angle $\psi$ - angle measured from the galaxy centre, between the plane and average line from centre to tips of outer isophotes (see Reshetnikov \& Combes 1998); position angle of average line passing through the tips of outer contour (measured from $\mathrm{N}$ to $\mathrm{E})$ - PA; direction of warp: clockwise $(+)$ or counter-clockwise $(-)$.

In the Appendix, we present the DSS images of all galaxies (in the $B_{\mathrm{J}}$ passband), rotated to horizontal.

\footnotetext{
${ }^{2}$ NASA/IPAC Extragalactic Database.
}

Figure 1 presents the distribution of the sample galaxies according to warp angle $\psi$. The distribution is truncated for $\psi \leq 4^{\circ}$ since we selected only galaxies with clearest warps to avoid selection effects. The mean value of $\psi$ is $4.8 \pm 1.3(\sigma)$ that is comparable with the amplitudes of optical warps found by Sanchez-Saavedra et al. (1990); Reshetnikov (1995), and de Grijs (1997). Dashed line in Fig. 1 shows the $\psi^{-5}$ law proposed by Reshetnikov \& Combes (1998) to fit the observed distribution. A naive extrapolation of this law to $\psi=0^{\circ}$ suggested that outer parts of all disk galaxies are warped with typical amplitudes of a few degrees.

In Fig. 2 we compare our measurements of the position angles of the sample galaxies with the FGCE data. The agreement is quite good. The mean difference is $<\mathrm{PA}-\mathrm{PA}(\mathrm{FGCE})>=-0.6 \pm 1.8$ (s.e.m.). Excluding two most deviating galaxies (FGCE 333, 981) we have $<\mathrm{PA}-\mathrm{PA}(\mathrm{FGCE})>=+0.6 \pm 0.4$ (s.e.m.).

It is evident in Figs. 3 that the projected spatial distribution of strongly warped galaxies and the distribution of their position angles are quite homogeneous (at least in the first order approximation). The large "void" in Figs. 3 is due to absorption in the plane of Milky Way. Comparison of the distributions for the galaxies with Sshaped and U-shaped warps shows that both distributions are statistically undistinguishable. There is no evidence of any significant large-scale alignment effect.

The number of galaxies with clockwise warps (18) is smaller than counter-clockwise galaxies (42). But, within our relatively poor statistics, the difference is not significant (both numbers are consistent within $3 \sigma$ ).

Reshetnikov (1995) found that disks of more massive and luminous galaxies are somewhat less warped. Our present data do not show any significant correlation (see Fig. 4).

\section{The environment}

Among the warped objects, 10 galaxies are members of interacting systems. The relative fraction of interacting galaxies $-17 \%$ - is higher than the analogous fraction $6 \%$ - for our complete sample of 540 galaxies (Reshetnikov \& Combes 1998). The fraction of isolated galaxies (9 objects $-15 \%)$ is smaller in the warped sample than in the control sample $(25 \%)$ while the relative number of galaxies with companions $(68 \%)$ is the same in both samples. This supports our conclusion that S-shaped warps are connected with galaxy environment (Reshetnikov \& Combes 1998). But this connection is not perfectly tight since there are warped galaxies among relatively isolated objects (an interpretation could be in terms of recent accretion).

To get more insight on the large-scale environment of warped galaxies, we have tried to compute the average 
Table 1. General characteristics of the sample galaxies

\begin{tabular}{|c|c|c|c|c|c|c|c|c|c|c|c|c|}
\hline FGCE & PGC & $\mathrm{ESO}$ & $\alpha(1950)$ & $\delta(1950)$ & $B$ & $\begin{array}{l}V_{\mathrm{r}} \\
\mathrm{km} \mathrm{s}^{-1}\end{array}$ & $\begin{array}{l}a \\
(') \\
\end{array}$ & $\begin{array}{l}b \\
(') \\
\end{array}$ & Type & $\begin{array}{l}\psi \\
\left({ }^{\circ}\right) \\
\end{array}$ & $\begin{array}{l}\mathrm{PA} \\
\left({ }^{\circ}\right)\end{array}$ & $\mathrm{D}$ \\
\hline 22 & & & 000856.5 & -324250 & & & 1.18 & 0.11 & $\mathrm{~d}$ & 3.5 & 178 & - \\
\hline 28 & 988 & 149-G 024 & 001221.0 & -560939 & 16.53 & & 1.29 & 0.15 & $\mathrm{bc}$ & 5 & 116 & - \\
\hline 38 & 1324 & 150-G 002 & 001815.0 & -564128 & 16.37 & & 1.12 & 0.15 & $\mathrm{bc}$ & 4 & 162 & - \\
\hline 44 & & & 002036.8 & -252730 & & & 1.03 & 0.13 & $\mathrm{c}$ & 4.5 & 40 & - \\
\hline 50 & & 294-IG 011 & 002459.7 & -405256 & 17.28 & & 1.34 & 0.19 & $\mathrm{~cd}$ & 4 & 44 & - \\
\hline 53 & 1729 & 112-G 004 & 002543.4 & -582248 & 15.86 & & 1.49 & 0.20 & $\mathrm{c}$ & 4 & 30 & - \\
\hline 63 & 2167 & 540-G 004 & 003343.4 & -192421 & 17.08 & & 1.10 & 0.10 & $\mathrm{c}$ & 5 & 131 & - \\
\hline 72 & & & 003925.8 & -331436 & 15.5 & 9525 & 1.05 & 0.11 & $\mathrm{bc}$ & 4 & 137 & - \\
\hline 80 & & 194-IG 037 & 004424.4 & -511546 & 17.11 & & 1.14 & 0.11 & $\mathrm{c}$ & 8.5 & 5 & - \\
\hline 99 & 3088 & 474-G 035 & 005014.9 & -260019 & 16.75 & & 1.12 & 0.13 & $\mathrm{bc}$ & 4 & 82 & + \\
\hline 108 & 3369 & 151-G 008 & 005427.5 & -553626 & 16.23 & & 1.23 & 0.13 & $\mathrm{~cd}$ & 3.5 & 86 & + \\
\hline 129 & 4010 & 113-G 013 & 010545.6 & -582034 & 16.62 & 5914 & 1.25 & 0.15 & $\mathrm{~cd}$ & 6 & 26 & - \\
\hline 170 & & & 013031.7 & -560738 & & & 1.29 & 0.11 & $\mathrm{c}$ & 4 & 112 & + \\
\hline 187 & 6349 & 477-G 001 & 014117.0 & -271159 & 16.82 & & 1.75 & 0.11 & $\mathrm{~cd}$ & 4 & 176 & - \\
\hline 189 & 6394 & 297-G 024 & 014229.0 & -404912 & 15.91 & 10171 & 1.26 & 0.18 & $\mathrm{bc}$ & 5 & 36 & - \\
\hline 202 & 6917 & 354-G 005 & 014953.7 & -334634 & 15.95 & 8703 & 1.70 & 0.22 & $\mathrm{bc}$ & 3.5 & 129 & + \\
\hline 226 & 8499 & 053-G 002 & 021225.7 & -710846 & 16.21 & 8000 & 1.88 & 0.20 & $\mathrm{~cd}$ & 3.5 & 109 & + \\
\hline 238 & 9125 & 355-G 014 & 022218.7 & -364721 & 17.37 & & 1.19 & 0.11 & $\mathrm{c}$ & 4 & 82 & + \\
\hline 240 & 9191 & 115-G 011 & 022346.2 & -575647 & 16.16 & & 1.68 & 0.20 & $\mathrm{c}$ & 5 & 14 & - \\
\hline 260 & & & 023939.4 & -331004 & & & 1.01 & 0.13 & $\mathrm{dm}$ & 4.5 & 25 & - \\
\hline 267 & 10605 & 299-G 017 & 024622.0 & -415137 & 16.74 & 20082 & 1.85 & 0.22 & $\mathrm{~b}$ & 6.5 & 39 & + \\
\hline 269 & 10640 & $356-\mathrm{G} 012$ & 024643.0 & -364324 & 15.82 & & 1.57 & 0.22 & $\mathrm{bc}$ & 4 & 35 & + \\
\hline 294 & & & 030320.5 & -414229 & & & 1.62 & 0.11 & $\mathrm{bc}$ & 9 & 132 & - \\
\hline 319 & 12791 & 116-G 019 & 032407.3 & -574031 & 16.1 & & 1.23 & 0.12 & $\mathrm{c}$ & 5.5 & 71 & + \\
\hline 320 & & & 032409.0 & -480840 & & & 1.12 & 0.11 & $\mathrm{c}$ & 5 & 131 & - \\
\hline 333 & & 482-G 005 & 033051.9 & -241806 & 15.40 & 1915 & 1.68 & 0.21 & $\mathrm{~cd}$ & 7 & 77 & + \\
\hline 354 & 13939 & 359-G 001 & 034814.8 & -345413 & 16.7 & & 1.74 & 0.20 & $\mathrm{c}$ & 3.5 & 128 & - \\
\hline 363 & 14212 & 249-G 035 & 035721.8 & -455956 & 16.24 & 1031 & 1.90 & 0.20 & $\mathrm{c}$ & 4 & 99 & - \\
\hline 377 & & & 040650.5 & -621050 & 16 & & 1.08 & 0.13 & $\mathrm{~cd}$ & 4 & 112 & - \\
\hline 382 & 14701 & 157-G 010 & 041237.5 & -541146 & 16.21 & & 1.21 & 0.13 & $\mathrm{c}$ & 4 & 83 & - \\
\hline 412 & & & 043010.7 & -293328 & & & 1.14 & 0.11 & $\mathrm{c}$ & 4 & 63 & - \\
\hline 416 & 15621 & 304-G 003 & 043435.3 & -375320 & 17.43 & & 1.18 & 0.12 & $\mathrm{~cd}$ & 3 & 42 & + \\
\hline 441 & 16116 & 361-G 012 & 044803.9 & -361118 & 15.14 & 5303 & 1.90 & 0.24 & $\mathrm{c}$ & 4 & 142 & - \\
\hline 539 & 17581 & 004-G 021 & 054910.1 & -842107 & 17.29 & & 1.12 & 0.12 & $\mathrm{c}$ & 3.5 & 170 & - \\
\hline 541 & 18052 & 364-G 010 & 055146.0 & -345636 & 16.39 & & 1.19 & 0.17 & $\mathrm{c}$ & 5 & 156 & + \\
\hline 623 & 19629 & 207-G 001 & 064641.1 & -481415 & 16.85 & & 1.01 & 0.12 & $\mathrm{~cd}$ & 3.5 & 139 & - \\
\hline 630 & 19816 & 087-G 050 & 065333.9 & -630919 & 15.77 & 3538 & 1.79 & 0.22 & $\mathrm{c}$ & 4.5 & 119 & - \\
\hline 638 & 20010 & 034-G 015 & 070321.8 & -772442 & 16.86 & & 1.34 & 0.12 & $\mathrm{c}$ & 5.5 & 18 & - \\
\hline 642 & & & 070937.3 & -511738 & & 19556 & 1.23 & 0.11 & $\mathrm{c}$ & 5 & 105 & + \\
\hline 674 & 21690 & $123-\mathrm{G} 023$ & 074341.6 & -580152 & 14.92 & 2920 & 2.91 & 0.34 & $\mathrm{~cd}$ & 4.5 & 107 & - \\
\hline 690 & 22797 & 059-G 026 & 080725.6 & -702047 & 16.81 & & 1.29 & 0.17 & $\mathrm{~b}$ & 4 & 179 & + \\
\hline 706 & 24027 & 562-G 017 & 083134.0 & -185200 & & & 1.01 & 0.10 & $\mathrm{c}$ & 6 & 154 & + \\
\hline 725 & 25300 & 496-G 025 & 085820.3 & -250215 & 16.97 & 4677 & 1.85 & 0.20 & $\mathrm{c}$ & 10 & 63 & - \\
\hline 806 & 30030 & $567-\mathrm{G} 038$ & 101435.3 & -210202 & 17.09 & & 1.12 & 0.13 & $\mathrm{bc}$ & 4 & 139 & - \\
\hline 834 & 31981 & 437-G 054 & 104117.5 & -283609 & 14.95 & 3461 & 1.90 & 0.27 & $\mathrm{~b}$ & 5 & 54 & - \\
\hline 835 & 32100 & 569-G 003 & 104256.4 & -222352 & 15.81 & 3731 & 1.59 & 0.22 & $\mathrm{dm}$ & 4.5 & 96 & - \\
\hline 840 & 32162 & 006-G 008 & 104654.8 & -833452 & 17.28 & & 1.57 & 0.15 & $\mathrm{c}$ & 3.5 & 135 & + \\
\hline
\end{tabular}


Table 1. continued

\begin{tabular}{|c|c|c|c|c|c|c|c|c|c|c|c|c|}
\hline FGCE & PGC & $\mathrm{ESO}$ & $\alpha(1950)$ & $\delta(1950)$ & $B$ & $\begin{array}{l}V_{\mathrm{r}} \\
\mathrm{km} \mathrm{s}^{-1}\end{array}$ & $\begin{array}{l}a \\
\left({ }^{\prime}\right)\end{array}$ & $\begin{array}{l}b \\
(')\end{array}$ & Type & $\begin{array}{l}\psi \\
\left({ }^{\circ}\right)\end{array}$ & $\begin{array}{l}\mathrm{PA} \\
\left({ }^{\circ}\right)\end{array}$ & $\mathrm{D}$ \\
\hline 871 & 33906 & $215-$ G 029 & 110807.1 & -475340 & 16.35 & & 1.12 & 0.16 & $\mathrm{~cd}$ & 4.5 & 12 & - \\
\hline 872 & & & 110857.1 & -420316 & & & 1.01 & 0.10 & $\mathrm{c}$ & 5 & 162 & - \\
\hline 891 & & & 112731.4 & -352925 & & & 1.01 & 0.09 & $\mathrm{c}$ & 4 & 64 & - \\
\hline 919 & 37906 & 505-G 003 & 115832.9 & -241730 & 14.10 & 1808 & 3.00 & 0.39 & $\mathrm{~m}$ & 4.5 & 132 & - \\
\hline 930 & & & 120617.6 & -353945 & & & 1.18 & 0.11 & $\mathrm{c}$ & 5 & 78 & - \\
\hline 944 & 39238 & 321-G 017 & 121310.2 & -422753 & 15.93 & 6704 & 1.70 & 0.24 & $\mathrm{~b}$ & 5.5 & 136 & - \\
\hline 974 & 42066 & 442-G 012 & 123355.0 & -275400 & 16.99 & & 1.01 & 0.13 & $\mathrm{c}$ & 6 & 33 & + \\
\hline 981 & & 381-G 014 & 124126.9 & -361412 & 15.15 & 3305 & 1.34 & 0.18 & $\mathrm{c}$ & 6 & 9 & - \\
\hline 1035 & & & 131215.4 & -254119 & & 13760 & 1.57 & 0.11 & $\mathrm{c}$ & 7 & 73 & - \\
\hline 1063 & & & 132355.3 & -505726 & & & 1.01 & 0.11 & $\mathrm{c}$ & 4.5 & 113 & + \\
\hline 1082 & & & 133228.4 & -365431 & & & 1.23 & 0.11 & $\mathrm{c}$ & 4.5 & 105 & - \\
\hline 1102 & & & 134537.9 & -462626 & & & 1.10 & 0.13 & $\mathrm{c}$ & 4 & 138 & - \\
\hline 1112 & 49478 & 445-G 077 & 135212.6 & -322647 & 17.27 & & 1.03 & 0.13 & $\mathrm{c}$ & 4 & 144 & - \\
\hline
\end{tabular}
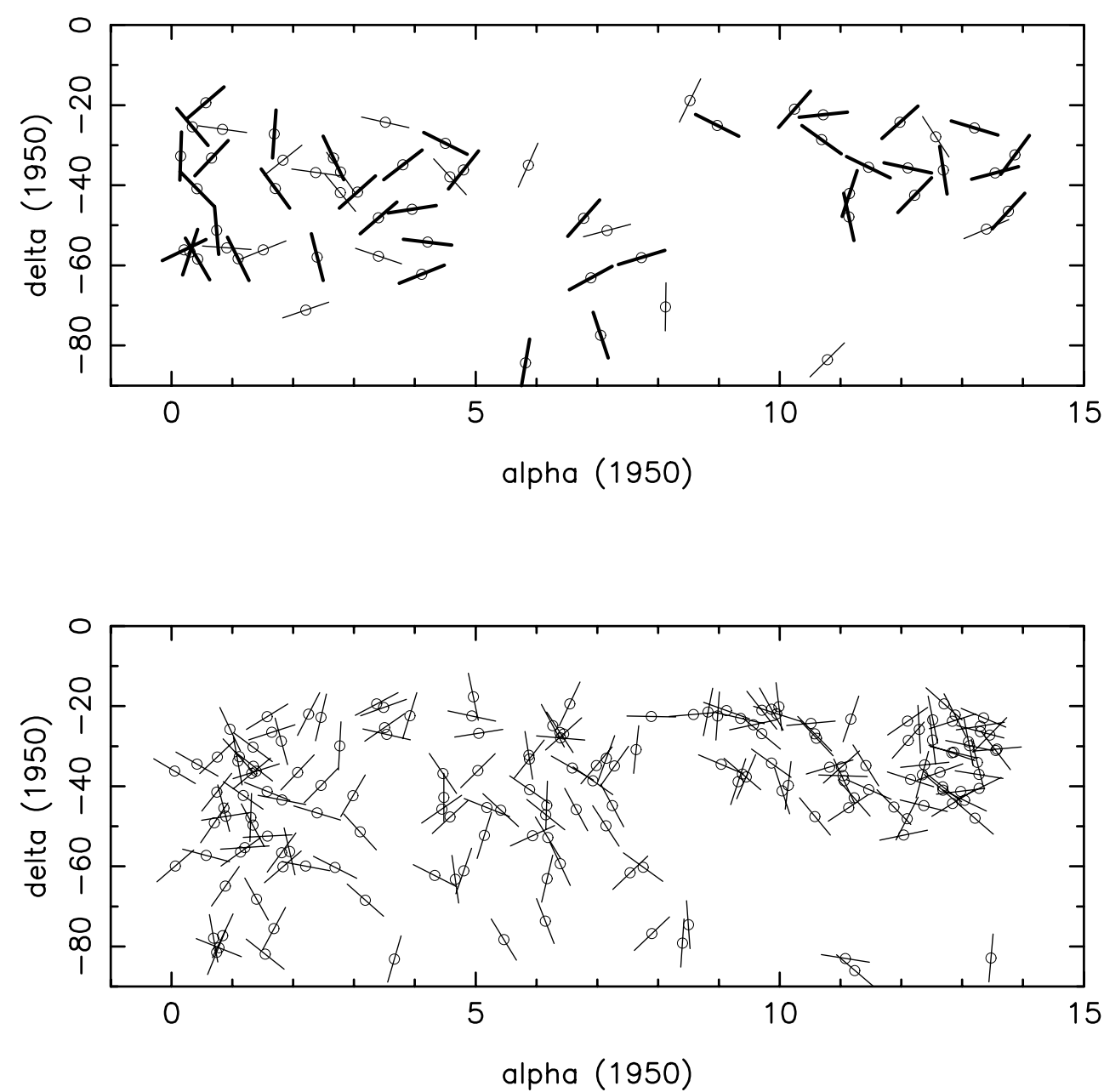

Fig. 3. Distribution of the warped galaxies on the sky: top - S-shaped warps, bottom - U-shaped warps. Plots of declinations (in degrees) versus right ascension (hours). The position angles of the galaxies (PA) are indicated by dashes (the length of each dash is $12^{\circ}$ ). Thick dashes show galaxies with counter-clockwise warp, thin dashes - clockwise warps (for S-shape warped galaxies) 
Table 2. Average density around warped and non-warped galaxies

\begin{tabular}{lccccc}
\hline$R_{\mathrm{s}}$ in Mpc & 10 & 15 & 20 & 25 & 30 \\
\hline Warped galaxies & $7.4 \mathrm{E}-2$ & $4.2 \mathrm{E}-2$ & $3.0 \mathrm{E}-2$ & $1.6 \mathrm{E}-2$ & $1.0 \mathrm{E}-2$ \\
Un-warped sample & $1.2 \mathrm{E}-2$ & $8.8 \mathrm{E}-3$ & $7.3 \mathrm{E}-3$ & $5.5 \mathrm{E}-3$ & $4.4 \mathrm{E}-3$ \\
\hline
\end{tabular}

$R_{\mathrm{s}}$ is the search radius, and the average densities are in gal $\mathrm{Mpc}^{-3}$

density of galaxies around the S-shape warped population, and compare it with a control sample. The control has been selected from the un-warped FGCE galaxies, with the condition that the asymmetry index along the minor axis is lower than 1.05 (cf. Reshetnikov \& Combes 1998). We have used the Southern Sky Redshift Survey (SSRS2, da Costa et al. 1998), where redshifts and magnitudes are reported for 5369 galaxies. Unfortunately, the redshifts are not known for all of the FGCE catalog, and we have only extracted from NED 15 redshifts for the warped sample, and 17 for the control (non-warped) galaxies. Some of them are plotted in Fig. 5 superposed on the SSRS2 points.

Around each object of our sample, we compute the average distance of the SSRS2 galaxies, given a search radius $R_{\mathrm{s}}$. This average distance $d_{\mathrm{m}}$ is computed taking the luminosity of galaxies as weight. The mean density is then estimated as

$\rho_{\mathrm{m}}=\frac{3 N_{\mathrm{gal}}}{4 \pi d_{\mathrm{m}}^{3}}$

where $N_{\text {gal }}$ is the total number of objects within $R_{\mathrm{s}}$. Taking a common weight for all galaxies only changes $d_{\mathrm{m}}$ by $10 \%$ at most. The results obtained for the warped and control samples are displayed in Table 2. The average density appears $3-4$ times higher for the warped objects. This result has to be confirmed by more statistics, when the redshifts for the whole FGCE catalog are known.

\section{Appendix}

We present here a condensed summary of the 60 warped galaxies photographs; each galaxy has been rotated by the position angle given in Table 1 and can be retrieved by its FGCE number.

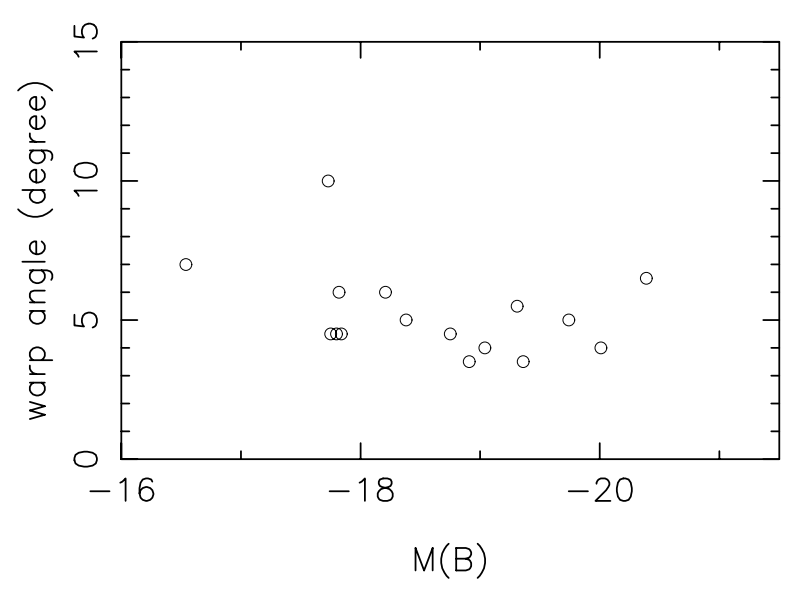

Fig. 4. Warp angle versus blue absolute magnitude for the Sshape warped galaxies $\left(H_{0}=75 \mathrm{~km} \mathrm{~s}^{-1} / \mathrm{Mpc}\right)$



Fig. 5. Location of some of the FGCE galaxies (filled triangles) among the SSRS2 survey objects (dots). The left sector corresponds to warped galaxies and right to un-warped ones, between declinations -24 and $-37^{\circ}$. Right ascensions run from 0 to $5 \mathrm{~h}$, and the maximum distance is $250 \mathrm{Mpc}$ $\left(H_{0}=75 \mathrm{~km} \mathrm{~s}^{-1} / \mathrm{Mpc}\right)$

\section{Conclusion}

In the present note we describe a new sample of southern spiral galaxies demonstrating strong S-shape optical warps. The galaxies were selected on the basis of their optical images from the DSS. First statistics indicate that warped morphologies are found preferentially in rich environment, although this result must be confirmed from larger redshift surveys. The sample gives the largest available material for future works (optical and $\mathrm{HI}$ ) on galaxy warps.

Acknowledgements. VR acknowledges support from Russian Foundation for Basic Research (98-02-18178), "Integration" programme (No. 578) and from French Ministère de la Recherche et de la Technologie. 


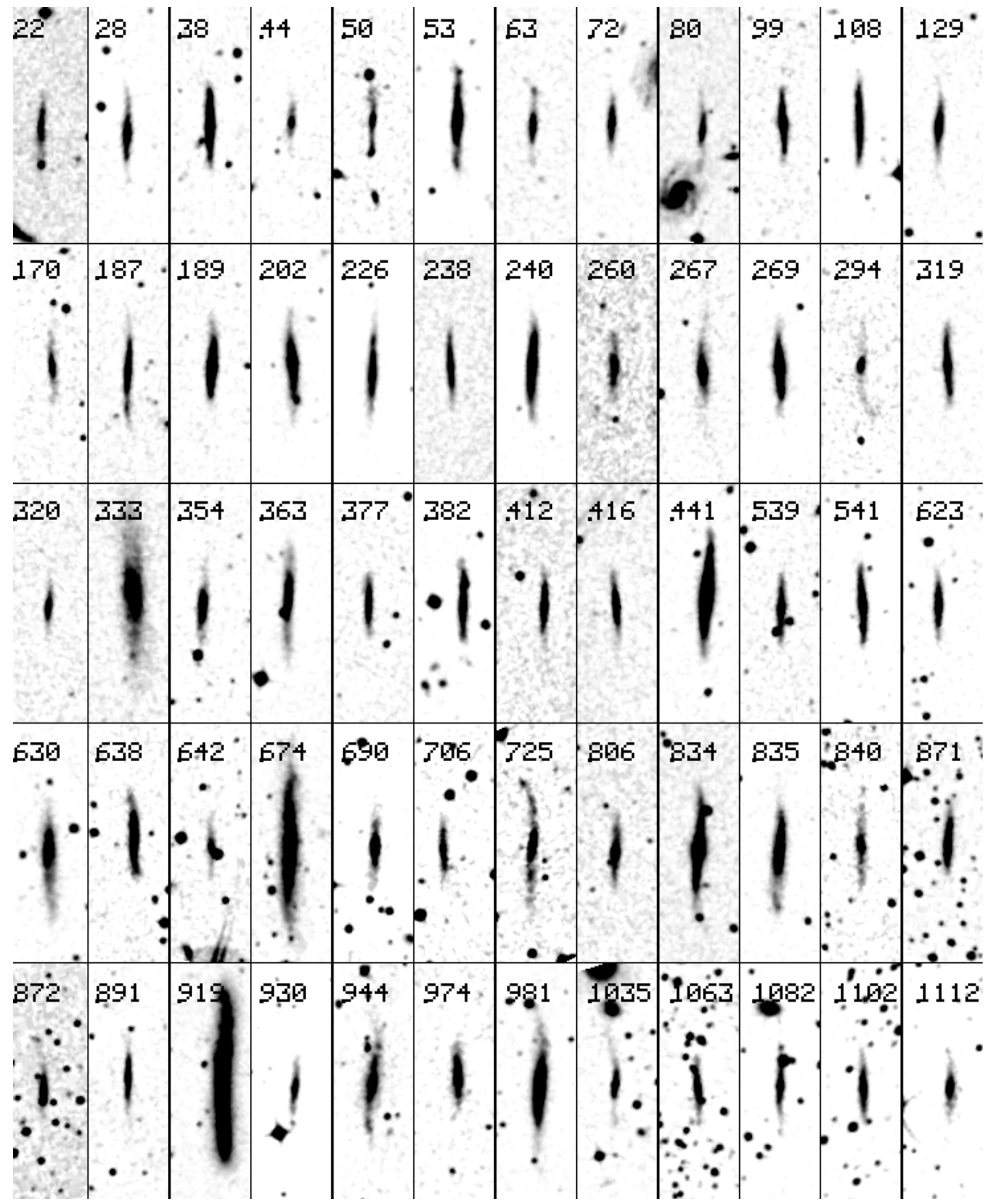

Fig. 6. Digital Sky Survey images of the S-shape warped galaxies. The size of each image is $1^{\prime} \times 3^{\prime}$. The images have been rotated, by -PA given in Table 1 


\section{References}

Binney J., 1992, ARA\&A 30, 51

Binney J., Jiang I.-G., Dutta S., 1998, MNRAS 297, 1237

Bosma A., 1981, AJ 86, 1791

Briggs F.H., 1990, ApJ 352, 15

da Costa L.N., Willmer C.N.A., Pellegrini P.S., et al., 1998, AJ 116,1

de Grijs R., 1997, Ph.D. Thesis, Groningen University

Dubinski J., Kuijken K., 1995, ApJ 442, 492

Hunter C., Toomre A., 1969, ApJ 157, 183

Kahn F.D., Woltjer L., 1959, ApJ 130, 705

Karachentsev I.D., Karachentseva V.E., Parnovsky S.L., 1993, Astron. Nachr. 314, 97 (FGC)
Lequeux J., Combes F., Dantel-Fort M., et al., 1998, A\&A 334, L9

New K.C.B., Tohline J.E., Frank J., Väth H.M., 1998, ApJ 503, 632

Ostriker E.C., Binney J.J., 1989, MNRAS 237, 785

Reshetnikov V.P., 1995, A\&AT 8, 31

Reshetnikov V., Combes F., 1998, A\&A 337, 9

Sanchez-Saavedra M.L., Battaner E., Florido E., 1990, MNRAS 246, 458

Sancisi R., 1976, A\&A 53, 159

Shang Z., Brinks E., Zheng Z., et al., 1998, ApJ 504, L23

Sparke L., 1984, MNRAS 211, 911

Sparke L., Casertano S., 1988, MNRAS 234, 873

Tubbs A.D., Sanders R.H., 1979, ApJ 230, 736 